definite association between complex spots which occur between 6.7 and $19.9^{\circ} \mathrm{E}$ on the solar disk and the occurrence of large geomagnetic disturbances on Earth. The mean displacement of these spot groups, $13.3^{\circ} \mathrm{E}$, can be taken as indicating that the spot groups and flares which cause the geomagnetic disturbances are located one day East of the solar meridian, allowing for their usual movement from East to West. For this particular sample, Horng also points out that $59.7 \%$ of the events occurred in the solar Northern Hemisphere below latitude $29^{\circ} \mathrm{N}$ and $40.3 \%$ in the Southern Hemisphere below latitude $25^{\circ} \mathrm{S}$. But that is hardly surprising since the entire period of the study was close to the most recent solar maximum, when sunspots always form at low latitudes.

\section{Image processing at Stanford}

\section{from Albert Macovski}

A meeting on "Image Processing for 2-D and 3-D Reconstruction from Projections: Theory and Practice in Medicine and the Physical Sciences", sponsored by Stanford University Institute for Electronics in Medicine and the Optical Society of America was held at Stanford University on August 4-7.

DuE primarily to the phenomenal success of the EMI brain scanner, the gencral subject of reconstruction from projections has generated a great deal of interest and enthusiasm. This particular meeting at Stanford, California is the second one of its type, the first taking place last year at the Brookhaven Laboratories.

Although X-ray and $\gamma$-ray imaging continue to dominate the scene, a number of novel application areas were presented which use comparable techniques. T. F. Budinger et al. (Lawrence Berkeley Laboratory) showed some preliminary results using heavy charged particles rather than $\mathrm{X}$ rays. Although the instrumentation is quite extensive, a significant dose reduction is obtained. J. F. Claerbout and P. S. Schultz (Stanford University) showed the application of similar techniques for seismic imaging using the reflections from sonic pulses. A cross-sectional image of the Earth is thus created to aid in activities such as oil exploration. J. F. Greenleaf, et al. (Mayo Foundation, Rochester) showed some exciting images of an excised dog's heart made by measuring the time-of-flight of ultrasonic pulses taken at many angles and positions. The reconstructed image is a cross section representing sonic velocity. A number of other interesting papers were presented on nonradiographic applications including nuclear magnetic resonance zengmatography, electron microscopy, radioastronomy and plasma diagnosis.

Many papers were devoted to the mathematics of reconstruction, both basic and related to specific applications. There was a strong trend, compared with the Brookhaven Conference, toward the use of direct as compared with iterative reconstruction procedures. In the basic area, R. P. Tewarson (SUNY, Stony Brook, New York) delighted the audience with his very profound fundamental treatment, well sprinkled with humour. He concentrated on methods for reconstructing the difference between the desired image and a standard image which he alluded to as the "average caucasian face." G. T. Herman et al. (SUNY, Buffalo), one of the pioneers of the iterative techniques, showed how these techniques converge to the least squares solution. Similarly P. R. Smith (Universitat Basel) along with T. M. Peters et al. (Christchurch Hospital, New Zealand) presented a fundamental exposition of the Fourier and back projection methods and the various aliasing considerations. M. Ein-Gal et al. (Stanford University) presented a decomposition of the projection data into consistent and inconsistent data and defined a quality factor representing their ratio. This talk invoked a lively discussion between the author, G. T. Herman and R. B. Marr of the Brookhaven Laboratory on the definition of inconsistency. $O$. $J$. Tretiak (Drexel Institute) presented an analysis of the point-spread function for direct reconstruction systems. This helped explain many of the artefacts which are being experienced.

In the area of mathematics related to specific applications a few investigators, including S. A. Johnson et al. (Mayo Foundation) discussed the problem of bending of rays due to refraction when reconstructions are made using optical or sonic waves. R. Gordon (National Institutes of Health), a pioneer in this field and the organiser of the meeting, presented a novel reconstruction system related to position reconstruction systems. Each event, as received, is assigned its most probable spatial position based on the previous events. A number of papers were presented on optimum reconstructions in the presence of incomplete data. L. R. D'Addario of the National Radio Astronomy Observatory and S. J. Wernecke (Stanford University) each presented papers on maximum entropy reconstructions. These gave impressive results in applications such as radioastronomy and electron microscopy, where the acquired data are sparse. A novel Fourier reconstruction system for nuclear medicine was presented by L. T. Chang et al. (Lawrence Berkeley Laboratory). A sequence of pinhole images were back projected and filtered in Fourier space to provide the desired reconstruction.

In the areas of improved X-ray and $\gamma$-ray reconstructions, the positron ring camera using coincidence techniques appeared quite exciting. L. Eriksson et al. (University of California, Los Angeles) and C. J. Thomson et al. (Montreal Neurological Institute) showed promising results. A number of papers were given on the problems of polychromatic effects in X-ray reconstruction. A. Macovski et al. (Stanford University) illustrated the nature of the distortion and a method of correction. R. E. Alvarez and Macovski (Stanford) showed how the X-ray spectrum could be decomposed so as to delineate density and atomic number effects in the reconstructed image. A similar decomposition based on chemical elements was presented by $\mathbf{M}$. J. Berggren et al. (Mayo Foundation). The fan beam configuration which allows simultaneous acquisition of Earth projection received considerable attention. D. P. Boyd (Stanford University) described a fan beam instrument using a xenon detector and $P$. Stonestrom et al. (Stanford University) discussed the scatter problems. The fan beam geometry presents some interesting mathematical reconstruction problems which were treated by R. King (General Electric, Schenectady) and L. Wang and L. H. Cho (University of California, Los Angeles).

\section{Phonon scattering in solids}

from a Correspondent

A conference on "Phonon Scattering in Solids" was held in Nottingham, UK on August 27-30. The proceedings will be published by Plenum Press.

Ar a good conference one finds out where the ragged ends in our knowledge lie. The conference recently held in Nottingham was no exception as contradictions were exposed by the close proximity of presented papers. and private conversations removed some of the gloss from published articles. But there were plenty of good new experiments reported which stimulated a lot of thought and the new techniques presented make it now possible to work at higher frequencies and with better spectral resolution. 